\title{
CORRIGENDUM
}

\section{Potent anti-obesity effect of enteric-coated lactoferrin: decrease in visceral fat accumulation in Japanese men and women with abdominal obesity after 8-week administration of enteric-coated lactoferrin tablets - CORRIGENDUM}

Tomoji Ono, Michiaki Murakoshi, Noriyuki Suzuki, Norio Iida, Motoyasu Ohdera, Masaaki Iigo, Toshihide Yoshida, Keikichi Sugiyama and Hoyoku Nishino

(First published online 22 May 2015)

doi:10.1017/S0007114510002734, Published by Cambridge University Press, December 2010.

The following information is added to the above publication in order to clarify a possible conflict of interest of the first authors with the funding organisation and the role of the funding organisation in the design and execution of the study.

\section{Conflict of interest declaration}

In the published article it was stated; 'The authors have no conflict of interest associated with the present study.' We wish to correct this statement to; 'T. O., M. M., N. S., N. I., M. O. and K. S. are employees of the Lion Corporation which provided financial support for this study.'

\section{Contribution of the funding organisation declaration}

In the published article it was stated; 'This research received no specific grant from any funding agency in the public, commercial or not-for-profit sectors.' We wish to correct this statement to; 'The Lion Corporation provided financial support for the study, but did not contribute in any other way to conduct of the study.'

\section{Reference}

1. Ono T, Murakoshi M, Suzuki N, et al. (2010) Potent anti-obesity effect of enteric-coated lactoferrin: decrease in visceral fat accumulation in Japanese men and women with abdominal obesity after 8-week administration of enteric-coated lactoferrin tablets. BrJ Nutr 104, 1688-1695, Published by Cambridge University Press, December 2010. 